\title{
Hacia la construcción de la voz critica de los estudiantes: etapas funcionales y roles de voz en la reseña de divulgación cientifica
}

\author{
An exploration of the resources for constructing a critical voice by \\ students: functional stages and voice roles in scientific reviews \\ Laura Aurora Hernández Ramírez \\ Universidad Autónoma de Tlaxcala
}

Resúmen: Comúnmente se considera que la evaluación ideal del conocimiento en el ámbito escolar es mediante la escritura de una opinión coherente sobre un tema revisado específico. Sin embargo, casi nunca se explica con precisión qué es opinar, ni el proceso por medio del cual el discurso configura las opiniones disciplinares esperadas. Como una introducción al análisis discursivo de estas formas disciplinares de la opinión, que representa una nueva veta de investigación dentro del análisis del discurso, en este artículo mostraré el tipo de recursos de posicionamiento autoral utilizados una serie de reseñas de divulgación científica (review) publicadas en la revista ¿cómoves?, editada por la UNAM. Concretamente, describiré los roles de voz (HOOD, 2004; MARTIN; WHITE, 2005; OTEÍZA, 2009) y sus estrategias de apertura y contracción del espacio dialógico con las voces participantes, que se definen en cada una de las etapas de estructura esquemática o secuencias a través de las cuales se cumple la función de recomendar la lectura de un libro relacionado con la ciencia. Para la descripción de estos recursos, empleo el sistema de categorías propuestas por la Teoría de la Valoración, un desarrollo del componente interpersonal que ha despertado gran interés en los investigadores y analistas discursivos de la Lingüística Sistémico Funcional, debido a su gran poder explicativo de la manera en que con el lenguaje se valora la experiencia social (OTEÍZA, 2009). Considero que a 
partir de este tipo de descripciones será posible proporcionar respuestas más precisas para las recurrentes interrogantes planteadas acerca de las convenciones de escritura de las diversas comunidades disciplinares y dará pie para la construcción de proyectos de intervención pedagógica de la argumentación especializada.

Palabras clave: Géneros de respuesta, reseña, opinión, discursivización, voz autorial

Laura Aurora

Hernández

Ramírez

Abstract: It is commonly considered that the ideal way to evaluate knowledge in the school context is by means of written coherent opinions about specific topics. However, teachers seldom explain with precision what it is to express an opinion, or the process through which discourse configures the expected disciplinary views. As an introduction to the discourse analysis of these disciplinary forms of opinion, which represents a new line of research, in this article I will show the types of authorial stance resources used in a series of scientific reviews published in the ¿Cómo Ves? magazine, edited by the National Autonomous University of Mexico. Concretely, I will describe the voice roles (HOOD, 2004; MARTIN; WHITE, 2005; OTEÍZA, 2009) and the expansion/contraction of dialogic space with the participant voices, which are defined in each of the stages of the schematic structure or sequence by which the function of recommending a science book is fulfilled. For the description of these resources, I use the system of categories proposed by Appraisal Theory, a development of the interpersonal component that has attracted great interest in discourse analysts and researchers from Systemic-Functional Linguistics because of its great explanatory power of the way in which social experience is evaluated with language (OTEÍZA, 2009). I believe that with this kinds of description it will be possible to provide more precise answers to the recurrent questions posed on writing conventions of the different disciplinary communities and to build up pedagogical projects about specialized argumentation.

Keywords: answer genres, review, opinion, discourse, authorial voice.

\section{Literacidad y opinión}

En los últimos 15 años, la bibliografía sobre enseñanza de lectura ha documentado ampliamente un enfoque discursivo de este proceso; en ella, han expuesto los expertos una gran cantidad de estrategias a través de las cuales un lector estratégico reconstruye el esquema conceptual planteado por un texto y se posiciona críticamente ante él (MARTÍNEZ, 2002a y b; SOLÉ, 2001, 2002). No obstante, cuando la tarea posterior a la comprensión tiene que ver con la de "expresar una opinión", los criterios de evaluación suelen plantearse a nivel muy general (sea preciso, sea crítico...) y/o limitarse a correcciones ortográficas y de formato. Durante el proceso, el estudiante suele tener dificultades para concretarla 
no sólo en términos de coherencia y cohesión, sino también para adecuarla discursivamente a las modalidades argumentativas en las que se construyen los conocimientos disciplinares específicos.

Es necesario tomar en consideración que el dar una opinión acerca de un tema en la lectura de estudio o académica es un proceso complejo que abarca tres fases: para empezar debe reconocerse el esquema conceptual planteado en el texto origen; después, debe llevarse a cabo un posicionamiento del lector de acuerdo con diferentes niveles: los conocimientos previos, el nivel de dominio en el proceso de lectura de género al que pertenezca el texto leído, tipo del objetivo lector, etc. Finalmente, como parte de la contextualización para la que se realiza la tarea de comprensión, se define el género en el cual se convertirá la opinión que se está pidiendo. De esta forma, “discursivizar" la opinión, la

Hacia la construcción de la voz crítica de los estudiantes última de estas tres fases, es una etapa que requiere que el enunciador de la misma sea capaz de construir una voz autoral intencional y crítica adecuada en términos de género y registro con la tarea que se le pida.

Desde este punto de vista, la opinión debe dejar de ejercerse como una instrucción expedita y tendrá que ser concebida como una práctica social discursiva que forma parte de la literacidad académica $y$ al igual que otras, es necesario enseñarla mediante una metodología sistemática y teóricamente fundamentada dentro de un proyecto de intervención académica adecuado. Si bien esta enseñanza discursiva de la opinión se hace indispensable en todos los niveles educativos, es fundamental en los niveles superiores de educación, pues los estudiantes deben iniciarse en el manejo de los recursos simbólicos más utilizados dentro de la comunidad de práctica profesional a la que aspiran incorporarse como especialistas. Sin embargo, este proyecto requiere se cuente con una descripción más sistemática de los géneros más importantes en la vida social y en las profesiones en el mundo hispánico, misma que se encuentra en proceso de investigación (ALEGRÍA, 2009; BOLÍVAR, 2004; MARINKOVIC, 2005; PARODI, 2010).

\section{Géneros de respuesta en la Lingüística Sistémico Funcional} El concepto de polifonía de Voloshinov (1929 [1992]) y retomado por Bajtín (1982) ha dado pie al planteamiento del tema de la construcción de las posturas evaluativas en el discurso académico desde diversas perspectivas lingüísticas. Una de los trabajos más abundantes es el de Hyland (2001, 2005, 2008), quien ha explicado el tema de la postura y el 
Laura Aurora

Hernández

Ramírez

386

compromiso (Stance y Engagement) como un sistema de recursos retóricos relacionado con el poder de persuasión que poseen los textos académicos. En sus trabajos ha definido la frecuencia de algunos rasgos que se utilizan como recursos para construir estas instancias evaluativas, tales como los marcadores modales de actitud, de certeza y la automención, preguntas, directivas, entre otros.

Desde una perspectiva sociocognitiva de análisis crítico del discurso latinoamericano, Bolívar (2004), Bolívar, Beke y Shiro (2010) describen al posicionamiento como uno de los resultados "naturales" de considerar al discurso como un espacio dialógico de interacción. Asimismo, plantean un análisis integrador de diversos niveles (micro y macro, es decir, desde estructuras clausulares a estructuras globales) para explicar cómo diferentes recursos lingüísticos se utilizan para "mostrar el papel estructural de la evaluación" en el discurso disciplinar (BOLÍVAR, BEKE; SHIRO, 2010, p. 97).

Dentro de la Lingüística Sistémico Funcional (LSF), uno de los temas más destacados en el desarrollo de la teoría en los últimos 10 años, es la manera en que a través del discurso nos posicionamos ante las imágenes discursivas construidas de los lectores, de los objetos o fenómenos de los que hablamos y de otros o de los mismos autores. Ya sea desde el análisis del Tenor, en la teoría del Registro o desde la Teoría de la Valoración de la Escuela de Sydney el aspecto interpersonal de la teoría se ha revelado como el eje de desarrollo teórico de la semántica discursiva (LUKIN, 2010; OTEIZA, 2009; OTEIZA; PINTO, 2011). En esta teoría se distinguen tres sistemas de análisis: el sistema del Afecto, el sistema del Compromiso y el sistema de la Gradación (OTEIZA, 2009; KAPLAN, 2004; MARTIN; WHITE, 2005). En el segundo de estos sistemas, es decir, en el del compromiso, situamos el estudio de los recursos por medio de los cuales los autores se posicionan y establecen una perspectiva de negociación con otras voces en la construcción del significado en sus textos. En general, se reconocen en estos trabajos dos puntos de referencia en cuanto a las posibilidades de negociación: la monoglosia, como aquella en la que no presentan otras posturas o voces participantes $\mathrm{y}$, por lo tanto, se trata de una propuesta poco o nada negociable; y la heteroglosia, en la que los textos reconocen la existencia de voces y posturas alternativas y, por lo tanto, su rango de negociabilidad semántica es notable (KAPLAN, 2004). Una de las maneras utilizadas para abrir o cerrar el espacio de negociación de las voces en el discurso son los recursos de intravocalización y extravocalización (WHITE, 2005). 
La extravocalización es el recurso en que el autor introduce explícitamente las voces externas; se trata del típico discurso reproducido, citado o reportado a través de procesos verbales predominantemente, por ejemplo:

a) La Asociación Internacional para el Estudio del dolor define el dolor como "una experiencia sensorial y emocional desagradable..."

b) Su autora Lynn Margulis...nos asegura que los microorganismos también son el resultado de un proceso evolutivo...

Hacia la construcción de la voz

En cambio, en la intravocalización el autor asume la responsabilidad de las aseveraciones en el texto, incluso como si las aseveraciocrítica de los estudiantes nes correspondieran al reseñador, movimiento que lo identifica con la monoglosia (a); también puede colocarse como quien puede describir o interpretar las intenciones que las otras voces realizaron (b):

a) Millones de años después, la aparición de los primeros fotosintetizadores, generó una crisis de magnitud difícil de imaginar...

b) Sexo, drogas y biología (y un poco de rock and roll) desmiente la opinión popular de que existen temas con los que la ciencia no debe meterse;

Relacionado también con este sistema, se consolida el estudio de los Roles de Voz (HOOD, 2004; IEDEMA, FEEZ; WHITE, 1994, citado en WHITE, 2005), mediante los que se concreta una perspectiva de negociación en los enunciados. Es decir, el rol de voz construye una o varias imágenes autoriales a través de las diversas etapas funcionales del texto (voz de observador, de investigador, etc., por ejemplo (HOOD, 2004)). Desde esas posturas, el autor abre o cierra el espacio de la negociación semántica (expansión y contracción dialógica (MARTIN; WHITE, 2005) mediante estrategias de posicionamiento en las que señala cómo los papeles sociales que conforman el contrato de enunciación de cada uno de los géneros establecen alianzas o tipos de relaciones de fuerza (MARTÍNEZ, 2002a). En el caso de las reseñas analizadas, se establece que en ellas se evalúen y se expresen opiniones explícitas sobre otros textos y mediante estrategias de intravocalización y extravocalización, los autores construyen una imagen de legitimación y verosimilitud en las posturas propuestas.

De esta forma es como propongo que una de las maneras de acercamiento a la enseñanza de los géneros de opinión en la escuela pueda hacerse a través de la lectura de géneros de respuesta (Response Genres) (CHRISTIE; DEREWIANKA, 2008), investigados con fines de enseñanza de 
Laura Aurora

Hernández

Ramírez

388 la literacidad en la escuela desde la teoría del Género desde finales del siglo pasado (ROTHERY, 1996). Estos géneros son muy útiles en el contexto escolar, pues se utilizan como medios de evaluación con tendencia más cualitativa que cuantitativa. En Rothery (1996) y Martin; Rose (2008) se proponen cuatro géneros de respuesta:

1) Respuesta personal, definida como una respuesta emocional a un texto.

2) Reseña, la descripción y evaluación del contenido de un texto literario, musical o visual.

3) Interpretación del mensaje de un texto.

4) Respuesta crítica, el análisis de los valores culturales expuestos en un texto.

Cada uno de estos géneros representa un nivel cada vez más complejo de respuesta y, por ende, de opinión acerca de los textos que sirven como base para el ejercicio del reconocimiento o construcción de la opinión. Por ejemplo, en el currículum australiano de New South Wales (NSW), durante la educación primaria, en la primera etapa (1ำ y $2^{\circ}$. grados) trabajan en los cursos de Inglés (su lengua materna) la respuesta personal como la manera de expresar sus opiniones aún muy ligadas a la experiencia inmediata y del sentido común. En las etapas 2 y 3 ( $3^{\circ} .4^{\circ}$. y $5^{\circ} .6^{\circ}$. grados, respectivamente), trabajan con la reseña de diversos contextos sociales: literarios, musicales, e incluso de artículos científicos en la última etapa ${ }^{2}$. En ellas, los estudiantes se inician en la comprensión más consciente de cómo los textos cumplen acciones sociales reales para diferentes tipos de lectores y audiencias (FRENCH, 2001). Los elementos valorativos abarcan elementos relacionados con la estima social y comprenden mejor la estructura global del texto. La interpretación y la respuesta crítica se utilizan a partir de la 3ª etapa, así como en la educación secundaria y preparatoria, con diferentes niveles de complejidad en estructura, propósitos, audiencias y recursos lingüísticos y valorativos en su composición (ROTHERY; STENGLIN, 2000). Estos textos, los elaboran en diversas materias del currículo.

A partir de una revisión de los currículos y planes de estudio oficiales de educación básica, reformados en el 2006 y disponibles al público en el portal web de la $\mathrm{SEP}^{3}$, y de su intención expresa de estar construidos en torno al concepto de prácticas sociales, las actividades didácticas no siempre definen clara ni extensivamente las caracterís- 
ticas de los géneros incluidos en la unidad didáctica o proyecto. Por ejemplo, las actividades con textos orales o escritos aún obedecen a clasificaciones textualistas que pueden pasar por alto la complejidad en la construcción de los textos especializados que no se escriben en torno a una sola forma de modo discursivo (CALSAMIGLIA, H.; TUSÓN, 2002), ni es sistemática su distribución curricular al no señalar una secuencia lógica entre los géneros trabajados. Tampoco son descritos los rasgos discursivos, textuales o lexicogramaticales de los textos que representan las diferentes prácticas sociales (HERNÁNDEZ RAMÍREZ, 2010). En la escuela secundaria, por su parte, los géneros de respuesta sólo están relacionados con la lectura literaria de cuentos, lo que reduce significativamente las posibilidades ante las cuales en nuestro mundo social actual es posible tomar un posicionamiento consciente por parte de los estudiantes: lo político, lo religioso, lo científico, etc. Además, no se hacen recomendaciones a los maestros sobre la metodología más sistemática con la que explicar estos géneros en su práctica dentro del aula.

Dentro de la cultura científica escolar mexicana, la escritura de reseñas se ha divulgado debido al concurso "Para leer la Ciencia desde México" que la editorial el Fondo de Cultura Económica, la SEP y el CONACyT organizan desde 1988. En este certamen, los participantes pueden elaborar un resumen, una reseña crítica o un ensayo según su edad y nivel de escolaridad. Al parecer, el nivel de aceptación se ha ido incrementando desde su creación de manera que en la octava edición del concurso se recibieron 60 mil trabajos ${ }^{4}$. Asimismo, en la Revista de divulgación científica ¿cómoves?, editada por la UNAM, existen modelos de este género en la sección ¿quéleer?, en donde se publican reseñas de recomendación de libros de investigación científica en las ciencias exactas y sociales. Estas reseñas son las que se utilizaron como muestra de análisis; se tomaron como modelos del género debido a su distribución nacional y de ser una de las únicas publicaciones de corte científico dedicadas a un público adolescente, edad en la que se empieza a solicitar con mayor insistencia la construcción de una opinión. En el Anexo se muestra la transcripción del texto de una de ellas (RDC1).

Este tipo de trabajos se proponen como modelo para iniciar al estudiante en una tarea crítica de evaluación de lecturas, para sustituir al poco claro "reporte de lectura" que es muy popular cuando se asignan tareas de este tipo, en especial cuando no se trate de textos literarios. 
Es así como el objetivo del análisis que presentaré en el siguiente apartado será mostrar algunas de las estrategias argumentativas realizadas a través de las voces autoriales en estas reseñas.

\section{El discurso de la divulgación científica. Rasgos}

La divulgación científica se ha definido como un movimiento institucional mediante el cual se hacen accesibles al público en general los temas y descubrimientos de la ciencia especializada. Para algunos au-

Laura Aurora

Hernández

Ramírez

390 tores (SÁNCHEZ MORA, 2000), su discurso puede entenderse como una recreación "literaria" del discurso profesional, cuyo objetivo es que los lectores se encuentren con el conocimiento científico mediante un texto placentero y educativo. Desde el punto de vista discursivo, se trata de una recontextualización de los textos "dentro de la vida cotidiana de los pueblos" (ALEGRÍA, 2009, p.211) como una elaboración creativa contextualizada del lenguaje científico especializado según Berruecos (2011).

Así pues, la reseña es uno de los textos que conforman el continuum de formas de comunicación que se despliegan entre los pares especialistas y el público lego que integra este género que, como cualquier género de respuesta, debe cumplir exigencias narrativas, argumentativas y didácticas (JEANNERET (1994), citado en MARINKOVIC, 2005) para interesar al gran público en los temas y el ámbito de la investigación científica. En general, se le caracteriza como un género didáctico, pues su contrato de enunciación relaciona a expertos (autores de libros reseñados y escritores de la reseña) con no expertos (público en general y/o estudiantes); sin embargo, es posible caracterizarlo como un híbrido gracias a que participa también de lo mediático, por sus medios de difusión y lo científico (BERRUECOS, 2011, p. 249). Su objetivo concreto es resumir y valorar el contenido de otro texto (escrito por un experto), al cual se le otorga un valor de aportación dentro del campo disciplinario de las ciencias, incluyendo a las ciencias sociales y humanísticos, pues dentro de los libros reseñados en el corpus en el que se basó este análisis se encuentran temas antropológicos, sociológicos y filosóficos. En el caso concreto de la muestra de este corpus, los reseñadores son científicos que se han especializado en el área de divulgación y no son periodistas que asumen el tema científico como una especialización, lo cual podría conllevar algún tipo de diferencia en la construcción de la perspectiva. 
Halliday y Martin (1993) han definido al discurso de la ciencia como "un complejo edificio ideológico", construido y mantenido por el discurso mismo. Su gramática está caracterizada, según Martin (1993) por el rasgo de la Technicality, es decir, por preferir una estructura lexicogramatical de alta densidad semántica derivada de una construcción clausular muy nominalizada y definida a partir de la desagentivización de sus procesos. Por ello, para Harvey $(2005$, p.97) se trata de un discurso construido desde la perspectiva de un sujeto observante, basado en un desarrollo atemporal, es decir, no narrativo, en el que se tematiza y se le da valor argumental al objeto de estudio y/o a la actividad de investigación. Sus proposiciones se presentan como abiertas a la discusión y están sujetas a cuestionamiento, pues plantean una relación simétrica entre un enunciador y enunciatario que pertenecen a la misma comunidad disciplinar como expertos.

En cambio, el discurso de la divulgación, definido como discurso didáctico, busca involucrar al lector no especializado en el ámbito del conocimiento científico. Por ello, en su gramática predomina la perspectiva de un sujeto participante en un entorno construido básicamente a través de los procesos o aconteceres materiales y está ligado al mundo real (MUÑOZ, 1999); en él, los hechos científicos no están puestos a discusión y su tematización se construye en torno a la fuente de información y/o a la relación ciencia y sociedad (HARVEY, 2005, p.97). Se trata, entonces, de un contrato de mediación entre el ámbito especializado y el del sentido común, en el que el científico divulgador necesita convencer a su lector del carácter benéfico de la actividad y pensamiento científicos que han sido tradicionales en la concepción moderna de la ciencia (BERRUECOS, 2011).

Así bien, en el análisis realizado fue evidente que para lograr este “convencimiento" definido como la acción social propia del discurso divulgativo, el autor manipula el espacio de negociación del conocimiento mediante los recursos evaluativos no sólo de tipo explícitamente evaluativo (que provienen de los sistemas del afecto y/o de la gradación: forma parte inevitable, panorama muy enriquecedor, explica de manera clara..), sino de cómo posiciona a su voz con respecto a las otras voces que participan en el contrato de habla del género y de cómo abre o cierra el espacio de negociación con estas voces que se despliegan en el espacio enunciativo. Este último tipo de mecanismos es el que analizaremos en el siguiente apartado. 


\section{La reseña de divulgación científica. Etapas funcionales y roles de voz}

Como se mencionó anteriormente, para este trabajo se analizaron 5 reseñas como muestra de análisis (RDC1 a la 5), todas extraídas de la sección ¿quéleer? de la revista de divulgación científica ¿cómoves? En ellas se reconocieron los roles de voz que se despliegan, así como la definición del tipo de imágenes enunciativas configuradas y la dinámica de voces autorales en la apertura y cierre del espacio de negociación semiótica.

Laura Aurora

Hernández

Ramírez Para ello, en primer lugar se distinguieron las etapas funcionales de la estructura esquemática de las reseñas, es decir, qué tipo de secuencias funcionales caracterizan la acción social de reseñar desde la divulgación de la ciencia. Posteriormente, se definieron las voces autorales presentes en ellas y las funciones que cumplen. Por último, se interpretó el tipo de recursos de intra y extravocalización que estas voces utilizan y a partir de ello definir el tipo de compromiso que establecen, es decir, si se trata de posturas que tienden a la heteroglosia o a la monoglosia.

Así pues, la estructura esquemática funcional de estos discursos se caracteriza por señalar tres etapas: la primera es en la que el autor ubica el tema, relaciona al lector con el tema y su vida cotidiana, justifica la importancia de conocer esta perspectiva del tema y presenta el texto y al autor reseñados; a esta etapa se le ha llamado Contextualización $^{5}$. En la segunda, la Descripción, el autor señala la idea central de cada una de las secciones y resume el contenido del libro reseñado. En la última, recomienda el tema y justifica nuevamente su importancia. Es necesario resaltar que en estas reseñas siempre se hace una evaluación positiva o recomendación de los textos reseñados en la mencionada sección. A esta última etapa se le ha llamado Evaluación. En la Fig. 1 se ilustra esta organización esquemática:

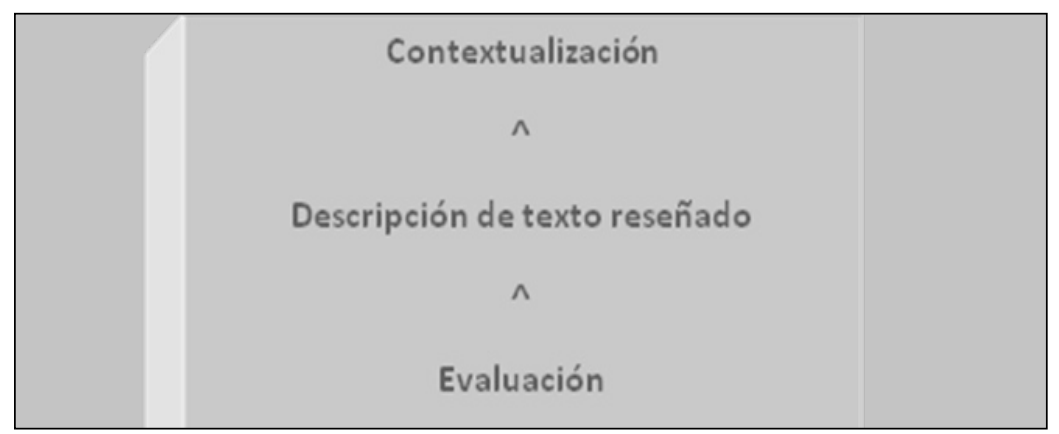

Fig. 1 Estructura esquemática de la Reseña de divulgación científica 
Como siguiente punto, es posible delinear que el enunciador se realiza en tres voces en las etapas funcionales señaladas: una voz de Explicador, el científico divulgador que se relaciona muy dinámica y abiertamente con el lector; una voz de Recopilador, que describe el contenido temático del textos y se mantiene a distancia del lector $\mathrm{y}$, finalmente, la voz del Evaluador, que representa al experto que recomienda y respalda las aportaciones científicas expuestas en el libro reseñado y el autor del mismo, y constituye la voz más monoglósica. Estas voces se van intercalando en las tres etapas antes mencionadas aunque puede observarse algún tipo de tendencia.

De esta forma, la voz del primero, a su vez, muestra dos vertientes cuyo cambio parece explicarse por la etapa en la que se ubica. En la contextualización o introducción de la reseña es en donde

Hacia la construcción de la voz crítica de los estudiantes el explicador muestra su mayor acercamiento con el lector pues es quien construye el sistema de alianza y relación entre los participantes. En general, construye un espacio en donde el mundo del sentido común se opone al mundo de la ciencia y los científicos (ejemplos 4 , 5 y 6) y en el cual él es el mediador que puede participar en ambos (ejemplos 1, 2 y 3):

1) Siempre que pienso en un hoyo negro recuerdo la secuencia del filme 2001: Odisea del espacio en la cual el astronauta.... RDC1

2) Para Jordi Agustí, el ajedrez se acerca más a la revolución biológica. RDC2

3) El objetivo de este libro destinado especialmente a los jóvenes es explicar qué es el dolor, qué lo causa y qué podemos hacer para aliviarlo... RDC4

4) Así plasma el cineasta su idea de esos objetos cósmicos llamados hoyos negros, cuyos secretos llaman la atención de científicos y no científicos. RDC1

5) Aunque la ciencia está presente en muchos aspectos de nuestra vida, la mayoría de las personas suelen percibirla como una actividad fría y distante. RDC5

6) Y si bien la comunidad científica hoy tiene más opciones que nunca para dar a conocer los avances de sus investigaciones, a menudo lo hace en términos que sólo pueden ser comprendidos por otros científicos. RDC5 


\section{Laura Aurora}

Hernández

Ramírez
Como es posible ver, este explicador utiliza el uso de deícticos personales de primera persona del plural (nosotros) para hacer alianza con el lector y el mundo cotidiano que comparten. El mundo de la ciencia (mencionada a través de frases nominales genéricas o nombres de los autores) se constituye en un tercero observado a través del explicador y mostrado desde una perspectiva conjunta al lector.

En una segunda fase de la contextualización, este explicador se acerca al mundo de la ciencia, mediante la definición de conceptos, temas o fenómenos relacionados con el tema central del texto reseñado. El reseñista cierra del espacio de negociación a través del uso de procesos más conductuales (usó...para sugerir que, ejemplo 7), hasta el uso de aseveraciones con procesos materiales, relacionales o existenciales que describen los fenómenos científicos (ejemplos del 8 al 11 en cursivas). Asimismo, la impersonalidad, uno de los rasgos más reconocidos del registro científico (HALLIDAY; MARTIN, 1993), se empieza a introducir en esta etapa dentro del espectro de opciones que identifican a las voces de los participantes en la interacción (elementos resaltados en negrita en los ejemplos 7, 8, 10 y 11):

7) Hawking usó la mecánica cuántica para sugerir que pese a la fama de tragarse todo, los HN podrían irradiar energía. RDC1

8) Los hoyos negros se forman al apagarse las estrellas más masivas luego de hacer explosión y derrumbarse sobre sí mismas en un proceso que se llama colapso gravitacional. RDC1

9) ... los organismos lo hacen (se mueven) en el espacio ecológico. $\mathrm{RDC} 2$

10) En ambos acasos, se puede llegar a niveles insospechados de complejidad y, sin embargo, toda la evolución como el ajedrez responde a reglas definitivamente sencillas. RDC2

11) Pero se piensa que hay otros, muy pequeños que pudieron formarse en los primeros instantes del Universo (hace unos 14000 millones de años). RDC1

En resumen, esta voz del explicador se relaciona con el desarrollo ideacional, pero al mismo tiempo asume una intermediación entre el campo disciplinar, pues forma parte de él, y es quien manipula el espacio de acercamiento de la ciencia con el lector. Su estrategia consiste en la intravocalización, hasta llegar a la monoglosia, pues por este medio 
se naturalizan los conocimientos que representan el conocimiento autorizado institucionalmente por la ciencia. Cabe aclarar que esta misma función en su vertiente más monoglósica se despliega, aunque en menor medida, en la etapa de la Descripción del texto (ejemplos 12 y 13):

12) ... y pronto surgieron los depredadores unicelulares. RDC1

13) Desde el punto de vista médico hay varios tipos de dolor: el nociceptivo, dividido en somático (en músculos, huesos, piel y mucosas) y visceral (en órganos internos)... RDC4

Hacia la construcción de la voz crítica de los

En una segunda voz, la voz del Recopilador, el autor se encarestudiantes

ga de señalar la organización textual. En la Contextualización esta voz cita o presenta a los autores, los títulos y la idea central de la obra que reseña, por lo que generalmente se trata de una opción heteroglósica (en negritas):

14) Un libro que da respuesta a múltiples preguntas sobre el tema es Los hoyos negros y la curvatura... de Shahen Hacyan, investigador del Inst. de Física de la UNAM. RDC1

15) Partiendo de esta metáfora, Jordi Agustí escribe El ajedrez de la vida, una reflexión sobre la idea del progreso en la evolución. RDC2.

En la Descripción es en donde esta voz predomina, pues con ella describe secuencialmente el contenido del texto; para ello, utiliza mayormente recursos de extravocalización citando literalmente o reportando la actividad verbal del autor del texto reseñado a través de procesos verbales, aunque sin mostrar acercamiento con el lector como lo hace la voz del explicador. Ejemplos:

16) “A medida que la masa del hoyo negro disminuye, su temperatura aumenta y la radiación se hace más intensa", escribe Hacyan. RDC1

17) Agustí empieza por exponer "las reglas del juego (...)" y "las fichas del juego (o el origen de las especies)". RDC2

18) ...y su autora comienza por preguntarse qué es la divulgación de la ciencia. La dificultad para responder -escribe- deriva en que no se trata de una disciplina... RDC3 
Laura Aurora

Hernández

Ramírez

19) María Antonieta Flores dedica unas páginas a la depresión; también a la anorexia, la bulimia y otros trastornos alimenticios. RDC4

20) El autor explica asimismo que estos misteriosos cuerpos producen una gravedad tan intensa... (RDC1)

21) Hacyan aborda también el tema de la radiación de Hawking, llamada así en honor del famoso físico inglés $\mathrm{SH}$, quien la propuso en 1974. RDC1

22) Desarrollando así su metáfora ajedrecística, Agustí va explicando los pormenores de la evolución. RDC2

Por último, el especialista crítico se realiza en la voz de Evaluador, voz que se encuentra en la Contextualización cuando justifica la importancia o pertinencia del tema del que trate el texto reseñado y se realiza como una voz monoglósica fundamentalmente que utiliza las aseveraciones con elementos modales de apreciación y gradación (subrayados) para expresar su punto de vista:

23) Ambas son indispensables para entender lo que es un agujero negro. RDC1

24) En ambos acasos, se puede llegar a niveles insospechados de complejidad y, sin embargo, toda la evolución como el ajedrez responde a reglas definitivamente sencillas. RDC2

25) Aunque se centra en la comunicación escrita de las ciencias naturales, las matemáticas y la tecnología en libros y revistas, es un texto tan bien documentado que abarca mucho más. RDC3

26) Ana María, maestra en física y literatura comparada, es por derecho propio una de las más reconocidas divulgadoras de la ciencia de nuestro país. RDC3

Esta voz domina en la Evaluación, la última de las etapas funcionales, y en estos casos siempre es positiva, es decir, siempre recomienda la lectura. En ella, utiliza recursos de intravocalización que llegan a la monoglosia para justificar la utilidad de la lectura y del conocimiento científico. De hecho, puede describirse como la etapa cuyo espacio dialógico está más cerrado hacia el lector y a otras voces porque aunque alude al lector (...parte de nuestra vida), el evaluador lo "obliga" a colocarse en la postura positiva en la que es colocada la ciencia, para lo 
cual utiliza elementos del sistema de la apreciación y de la gradación (manera clara y precisa, poética, nueva versión...) como en otras etapas evaluativas mostradas con anterioridad. El tipo de procesos utilizados varía notablemente pues utiliza ahora materiales (ofrece, convertirá), mentales (pensar) y relacionales (forma parte), pero predominan los conductuales (invita a imaginar, animando, seducir). Ejemplos:

27) Este libro invita al lector a imaginar qué sucede dentro de esos caníbales cósmicos. RDC1

28) Aunque las ideas científicas que presenta este libro varían en grado de complejidad, Agustí las explica de manera clara y a veces poética, animando así al lector que se acerca por primera vez a estos temas... RDC2.

Hacia la construcción de la voz crítica de los estudiantes

29) ... pero ofreciendo también esta nueva versión a los que se han dejado seducir por el estudio de la evolución biológica. RDC2

30) Este texto, con exquisitos tintes literarios y estupendos ejemplos, nos hace pensar en los objetivos de la divulgación de la ciencia y nos ofrece un panorama muy enriquecedor sobre esta labor, hoy indispensable. RDC3

31) Con seguridad se convertirá en una referencia obligada... para todos aquellos que disfrutan y aprenden con la divulgación de la ciencia... RDC3

32) y no sólo para aquellos que nos dedicamos a ella. RDC3

33) La obra termina con un glosario y una bibliografía sobre un tema que forma parte inevitable de nuestra vida. RDC4

A partir de estos datos es posible concluir que en la construcción del espacio de relaciones de posicionamiento de estas voces se definen dos áreas de negociación semiótica: una relaciona al reseñador con la comunidad discursiva de la ciencia, con la que hace explícito su acercamiento o no; a esta etapa se le llama movimiento retrospectivo (MARTIN; WHITE, 2005; KAPLAN, 2007) que en estos textos es la etapa heteroglósica, pues en donde diferentes voces participan en diversos grados de abertura. Asimismo, existe otra área de participación con los lectores o definida como movimiento anticipatorio, en donde el autor establece relaciones de alianza en el mundo de la vida cotidiana y como el que los acerca e involucra en el mundo de la ciencia. En la muestra analizada, este espacio se abre especialmente en la Contextualización o introduc- 
Laura Aurora

Hernández

Ramírez

398

ción de la reseña para involucrar al lector con el tema y el mundo de la ciencia, pero conforme se acerca al término el texto, el espacio se cierra a mostrar posturas alternativas al lector.

De esta forma, es posible definir al registro de este género como un discurso heteroglósico, porque en él convergen diversas voces: las creadas por el explicador, por el reseñador con sus diversos grados de acercamiento. La "amabilidad" del discurso se crea en estas instancias de posicionamiento interpersonal. Sin embargo, no se trata de una postura de negociación ideacional, la ciencia se proclama a través de voces autorizadas: la ciencia, el libro reseñado y su autor como parte de ella. Las voces autorales del reseñista son armónicas con la voz de la ciencia, representada en el autor del libro descrito. Es, por lo tanto, un discurso fuertemente monoglósico (HARVEY, 2005) con una "imagen heteroglósica", que sirve de medio para atraer la atención del enunciatario hacia la realidad científica.

Si bien el espacio dialógico se abre al mundo del sentido común -su contrario-, sólo lo hace como punto de referencia para conectar al lector lego con un mundo especializado, el cual se configura como un conocimiento benéfico, conveniente y ubicado en un estadio de mayor relevancia que el del sentido común. Este último es el recurso más recurrente para justificar la importancia de hablar del tema.

En resumen, el papel de reseñador es de un intermediario más experto que el lector, pero igual al autor del texto reseñado; es quien posee los conocimientos disciplinares para evaluar a otro experto en el área, pero al mismo tiempo, sabe relacionar ese conocimiento con el sentido común del lector lego por lo que se confirma el carácter didáctico del discurso de divulgación descrito por Marinkovic (2005).

En cuanto a los recursos lexicogramaticales de la opinión que ha sido el objetivo principal de análisis en este texto, es posible afirmar que las evaluaciones coinciden con las etapas más monoglósicas y coinciden con el uso de afirmaciones que explican en un registro tradicionalmente científico (expresiones relacionales e impersonales) o en expresiones con elementos evaluativos de apreciación y gradación más explícitos. El uso más heteroglósico de las voces más didácticas (voz del explicador y recopilador) emplea recursos de acercamiento con el lector por medio de deícticos personales, especialmente las primeras personas del singular y del plural (HYLAND, 2001). El recurso de extravocalización más recurrente es el discurso citado y reportado con procesos del decir para hacer participar a las voces especialistas que acompañan el desarrollo y descripción del texto reseñado. 


\section{Conclusiones o implicaciones para la enseñanza}

Para que la escritura escolar forme parte de una experiencia real y, por lo tanto, su aprendizaje en la escuela sea significativo y consistente, es necesario ligar los géneros de la vida social con los escolares. Es decir, hay que esforzarse por crear en los contextos escolares situaciones sociales congruentes con las situaciones sociales que los estudiantes pueden vivir fuera del contexto escolar, en su contexto profesional, laboral, pero también en el contexto ciudadano (político y cultural) del que irremediablemente forman parte y tendrán que constituirse en participantes más activos.

En este sentido se propone que la reseña y otros géneros de respuesta se conviertan en las maneras de describir, valorar, interpretar textos dentro de la escuela, sustituir las denominaciones va-

Hacia la construcción de la voz crítica de los estudiantes gas y poco precisas del "reporte de lectura" cuyas características dependen de un momento escolar muy particular y parece sólo tener un valor como instrumento de calificación poco cualitativa y significativa. El mostrar a los estudiantes el funcionamiento de estos rasgos de evaluación y posicionamiento de los autores, aunque en este caso sean todos ellos a favor de la ciencia, se considera un punto de partida para que tomen conciencia de cómo con el lenguaje se construyen las realidades, las posturas, las opiniones, etc. Los textos de revistas más especializadas podrían servir para mostrar cómo se construyen otro tipo de posicionamientos.

En la escuela mexicana aún es necesario trazar políticas más claras sobre el ejercicio de una literacidad que sea acorde a nuestras necesidades sociales, en un ejercicio que se "favorezca la inclusión...la interculturalidad y las necesidades educativas especiales con equidad" (MEXICO, 2011). Aún necesitamos encontrar rutas, la investigación sobre los géneros en nuestra cultura y su sistematización dentro de los currículos de enseñanza de lengua y de todas las áreas, puesto que la literacidad no es exclusiva de una asignatura, nos dará una mayor certidumbre en el desarrollo humano de nuestros ciudadanos.

A través del análisis de las estructuras de los géneros como el aquí descrito, así como sus rasgos semánticos, textuales y lexicogramaticales será posible comprender con mayor solidez las estrategias discursivas que los caracterizan. Este conocimiento no sólo es de utilidad en el reconocimiento del manejo ideológico de la información, sino en el conocimiento metadiscursivo que todo escritor aprendiz debe desar- 
rollar como parte de sus habilidades y a sus maestros nos servirá para mostrarles el camino de la construcción de la voz en el discurso como manera de explorar sus propias posibilidades en el desarrollo de sus voces discursivas.

\section{Referencias}

Laura Aurora

Hernández

Ramírez

400
ALEGRÍA, M. (Coord.). Cómo leer La Ciencia para Todos. Géneros discursivos. México: FCE, 2009.

BAJTÍN, M. Estética de la creación verbal. México: XXI, 1982.

BERRUECOS, M. de L. De agujeros negros a "Hoyos negros": la física en la historia ilustrada. En Anuario de investigación. México, UAM-Xochimilco, 2011. pp. 241 -274.

BOLÍVAR, A. Análisis crítico del discurso de los académicos. Rev. Signos, vol.37, n.55, 2004. pp. 7-18.

BOLÍVAR, A.; BEKE, R.; SHIRO, M. Marcas lingüísticas del posicionamiento en las disciplinas: Estructuras, voces y perspectivas discursivas. En PARODI, G. (Ed.) Alfabetización académica y profesional en el siglo XXI: Leer y escribir desde las disciplinas. Chile, Ariel, 2010. pp.95-125.

CALSAMIGLIA, H.; TUSÓN, A. Las cosas del decir. Barcelona, Ariel, 2002.

CHRISTIE, F.; DEREWIANKA, B. School Discourse. London: Continuum, 2008.

FRENCH, R.). Reading and writing reviews in Stage 2 and 3. In Scan 20(1), February, 2001. pp. 4-9. Consultado el 3 de marzo de 2012. Disponible en: http://www.curriculumsupport.education.nsw.gov.au/ schoollibraries/scan/content/scanarch2000-2001.htm

HALLIDAY, M.A.K.; MARTIN, J.R. Writing Science. Great Britain: The Falmer Press, 1993. 
HARVEY, A.M. Manifestaciones evaluativas en la ciencia como discurso. Un estudio comparativo. En HARVEY, A.M. (Comp.). En torno al discurso. Contribuciones de América Latina. Chile: Ediciones Universidad Católica de Chile, 2005. pp. 94-125).

HERNÁNDEZ RAMÍREZ, L.A. El Género como concepto organizador del currículum en el Programa 2006 de español en la Escuela Secundaria, en Memorias del XI Encuentro Nacional de Estudios en Lenguas, México: Universidad Autónoma de Tlaxcala, 2010. Disponible en: http://filosofia.uatx.mx/ponencias/cuarentayseis.pdf. 2010.

HYLAND, K. "Humble servants of the discipline? Self mention in research articles. In English for Specific Purposes, 20, 2001. pp. Hacia la construcción de la voz crítica de los estudiantes 207-226.

Stance and Engagement: a model in interaction in academic discourse. In Discourse Studies, 7(2), 2005. pp.173- 192.

Disciplinary Voices. Interactions in Research Writing. In Journal of English Text Construction, London: John Benjamin Publishing Company, 2008. pp.5-22.

HOOD, S. Apraising research: Taking a stance in academic writing. Tesis doctoral no publicada, Universidad Tecnológica de Sydney, Sydney, 2004.

KAPLAN, N.). Nuevos estudios en el estudio de la evaluación en el lenguaje: La Teoría de la Valoración. En Boletín de Lingüística, 22, julio-diciembre, 2004. pp. 52-78.

La construcción discursiva del evento conflictivo en las noticias por televisión. Tesis de doctorado no publicada, Facultad de Humanidades y Educación. Universidad Central de Venezuela, 2007.

LUKIN, A. News and Register: A Preliminary Investigation. En MOHBOOH, A.; KNIGHT, N. Appliable Linguistics. Great Britain: Continuum, 2010.pp. 92-112. 
MARINKOVIC, J. Los textos especializados en la educación media técnico-profesional. Hacia una clasificación en el ámbito de la divulgación científica. En HARVEY, A.M. (Comp.). En torno al discurso. Contribuciones de América Latina. Chile: Ediciones Universidad Católica de Chile, 2005. pp. 129-134.

MARTIN, J.R. Life as a noun: Arresting the Universe in Science and Humanities. In: HALLIDAY, M.A.K.; MARTIN, J.R. Writing science.

Laura Aurora Hernández

Ramírez 402 Great Britain: The Falmer Press, 1993. pp. 221-267.

MARTIN, J.R.; WHITE, P.R.R. The language of evaluation: appraisal in English. Great Britain: Palgrave Macmillan, 2005.

MARTIN, J.R.; ROSE, D. Genre relations. USA: Equinox, 2008.

MARTINEZ, M.C. Lectura y escritura de textos. Perspectivas teóricas y talleres. Cali: Universidad del Valle y Cátedra UNESCO MECEAL, 2002a.

. (Comp.) Propuesta de intervención pedagógica para la comprensión y producción de textos académicos. Cali: Universidad del Valle y Cátedra UNESCO MECEAL, 2002b.

MUÑOZ, N. I. La (des)agentivación en el lenguaje científico y en el de divulgación. En Anclajes. Revista del Instituto de Análisis semiótico del Discurso III. 3,1999. pp. 95-115.

OTEÍZA, T. Solidaridad ideológica en el discurso de la historia: Tensión entre orientaciones monoglósicas y heteroglósicas. En Revista Signos. Estudios de Lingüística 42(70), 2009. pp. 219-244.

OTEIZA, T.; PINTO, D. En (Re)Construcción: discurso, identidad y nación en los manuales escolares de historia y de ciencias sociales. Chile: Editorial Cuarto Propio, 2011.

PARODI, G. (Ed.) Alfabetización académica y profesional en el siglo XXI: Leer y escribir en las disciplinas. Chile: Ariel, 2010. 
ROTHERY, J. Making changes: developing an educational linguistics. In HASAN, R.; THOMPSON, G. (Ed.). Literacy in Society. London: Longman, 1996.

ROTHERY, J.; STENGLIN, M. Interpreting literature: the role of Appraisal. In Unsworth, L. Researching Language in Schools and Communities. London: Continuum, 2000. pp. 222-244.

Hacia la construcción

MEXICO. Secretaría de Educación Pública, Programa de Secundade la voz ria. México: SEP, 2011. crítica de los estudiantes

SÁNCHEZ MORA, A.M. La divulgación de la ciencia como literatura. México: Dirección General de Divulgación de la Ciencia, UNAM, 2000.

SOLÉ, I. Estrategias de lectura. Barcelona: Graó, 2001. De la lectura al aprendizaje. En Lomas, C. El aprendizaje de la comunicación en las aulas. España: Paidós, 2002. pp. 107-122. VOLOSHINOV, V. M. El marxismo y la filosofía del lenguaje. Trad.: T. Bubnova. España: Alianza Editorial, 1982 [1992].

WHITE, P.R.R. Un recorrido por la teoría de la valoración. Trad.: E. Ghío. En Appraisal Website: http://www.grammatics.com/ appraisal/. 2005. Website developed by WHITE, P.R.R. and ELDOn, Verbosity Enterprises. Recuperado: 7 de octubre de 2011.

\section{Notas finales:}

\footnotetext{
1. Los ejemplos son tomados de las reseñas de la muestra de análisis.

2. Estas etapas corresponden a las que organizan el plan K6 (Inglés) del currículum australiano. En los siguientes vínculos pueden consultarse los syllabus de los diferentes niveles escolares. En ellos, además se describen los objetivos y los logros educativos de educación preescolar y primaria. Cabe señalar que sus fundamentos se encuentran en la Pedagogía basada en el Género de las LSF: http:// www.curriculumsupport.education.nsw.gov.au/index.htm; http://www.boardofstudies.nsw.edu.au/k-6/; http:// k6.boardofstudies.nsw.edu.au/go/english

3. http://basica.sep.gob.mx/dgdc/sitio/start.php?act=rieb

4. Cfr. La página web del concurso: $h t t p: / / w w w . l a c i e n c i a p a r a t o d o s . m x / H i s t o r i a . a s p x$

5. Cfr. Anexo. Las secciones resaltadas señalan los límites de las tres etapas descritas.
} 
Anexo

\title{
Reseña RDC1
}

ÁVILA JIMÉnEZ, N. Los objetos más exóticos del universo. ¿Cómo ves?, 38. 2009.

\section{LOS OBJETOS MÁS EXÓTICOS DEL UNIVERSO}

\author{
Norma Ávila Jiménez
}

Laura Aurora

Hernández

Ramírez

404

Siempre que pienso en un hoyo negro recuerdo la secuencia del filme 2001: Odisea del espacio en la cual el astronauta Dave Bowman penetra asombrado en lo que Arthur C. Clarke llamara "la puerta de las estrellas". Acompañado por música de Gyorgy Ligeti, Bowman es tragado por un vórtice cósmico que lo traslada a otra región del espacio-tiempo. Stanley Kubrick, director de esta película y coautor, con Clarke, del guión, lleva a Bowman entre innumerables planos de colores mientras pareciera que su rostro se ha quedado congelado, como si el tiempo pasara muy despacio.

Así plasma el cineasta su idea de esos objetos cósmicos llamados hoyos negros, cuyos secretos llaman la atención de científicos y no científicos. Un libro que da respuesta a múltiples preguntas sobre el tema es Los hoyos negros y la curvatura del espacio-tiempo, de Shahen Hacyan, investigador del Instituto de Física de la UNAM. Hacyan, reconocido divulgador de la ciencia, explica de manera accesible la ley de la gravitación universal de Newton, así como los principios de [la] teoría general de la relatividad de Albert Einstein. Ambas son indispensables para entender lo que es un agujero negro. El autor explica asimismo que estos misteriosos cuerpos producen una gravedad tan intensa, que ni siquiera la luz podría escapar de su superficie. Los hoyos negros se forman al apagarse las estrellas más masivas luego de hacer explosión y derrumbarse sobre sí mismas en un proceso que se llama colapso gravitacional.

Hacyan aborda también el tema de la radiación de Hawking, llamada así en honor del famoso físico inglés Stephen Hawking, quien la propuso en 1974. Hawking usó la mecánica cuántica para sugerir que pese a su fama de tragarse todo, los hoyos negros podrían irradiar energía. Al hacerlo, tendrían que perder energía, o lo que es lo mismo en la teoría de la relatividad, perder masa. "A medida que la masa del hoyo negro disminuye, su temperatura aumenta y la radiación se hace más intensa", escribe Hacyan. "Consecuentemente, la masa disminuye cada 
vez más y más rápidamente, hasta que el hoyo negro se evapora totalmente en una verdadera explosión". El efecto Hawking es insignificante para los hoyos negros muy masivos que se forman por colapso gravitacional. Pero se piensa que hay otros, muy pequeños, que pudieron formarse en los primeros instantes del Universo (hace unos 14,000 millones de años). Esos "se encontrarían en la actualidad justamente en la etapa final de evaporación". Este libro invita al lector a imaginar qué sucede dentro de esos caníbales cósmicos.

Hacia la construcción de la voz crítica de los estudiantes 
